\title{
EDITORIAL
}

\section{Multiple team membership: current state of affairs and directions for future research}

\author{
NICOLETA MESLEC \\ Department of Organization Studies, Tilburg University, The Netherlands
}

\section{Introduction}

Organizations are operating in environments that are highly dynamic and are faced with the challenge to adapt such that they maintain and even further increase their levels of performance and innovativeness. As a form of adaptation, many organizations changed the way in which they organize their work around teams. Nowadays we see more and more that employees are part of multiple teams (instead of single teams) at the same time and need to contribute to various tasks simultaneously. Reports are indicating that $65-95 \%$ of knowledge workers belong to more than one team at the same time (O'Leary, Mortensen \& Woolley, 2011). This phenomena of being part of more than one team simultaneously has been defined as multiple-team membership (O'Leary et al. 2011). Other scholars such as Margolis (2019) have defined the concept in a broader way such as employees having membership interdependencies across teams.

Despite the fact that multiple team membership is widely used as a form of structuring work in organizations, empirical research on the topic has only recently started to emerge. The seminal theoretical work of O'Leary et al., (2011) has placed the concept on the research agendas of both Organization Behavior and Project Management fields. The review conducted by Margolis (2019) identified 44 research articles connected to the topic of multiple-team membership. The authors identified various antecedents, outcomes and experiences connected to the multiple team membership context. In terms of outcomes most research focused on productivity, learning, effectiveness and connections across teams. Oftentimes the idea of non-linear relations is being discussed given that being part of too many teams at the same time and doing too many (different) tasks simultaneously might interfere with effectiveness and learning. In terms of antecedents, research is less rich and has mainly looked at characteristics of an employee, ranking within organization and, status within teams (Cummings \& Haas, 2012). Finally, in terms of experiences, concepts such as team membership identification and empowering leadership have been examined (Rapp \& Mathieu, 2019; Chen et al., 2019).

\section{Research agenda in multiple- team membership}

To be noted that in the past multiple team membership was seen as a nuisance, that one needs to control for, in order to research single teams. Research conducted so far in the multiple-team membership field is promising and acknowledges that the concept needs to be studied as a self-standing concept in the Organization Behavior and Project Management fields. Although research in the area is growing and knowledge continues to 
accumulate, there are still many research questions that have remained unanswered. What is currently missing in the multiple-team membership field of research and what are good practices to study it? These are two questions that will be addressed in the next sub-sections.

\section{Multiple-team membership and leadership}

It is surprising to observe that we do not know much with respect to the topic of leadership in the multiple-team membership context. One exception is the study of Chen et al. (2019) where they examine the role of empowering leadership. In three different studies they identify that team leaders' empowering leadership influences in a positive way the employee's psychological empowerment and this leads to an increase in proactive behaviors. Next to empowering leadership, other existing forms of leadership might also be relevant to study in this context.

One type of leadership that might be particularly relevant in the context of multipleteam membership is the boundary spanning leader. Boundary spanning behaviors are extremely relevant especially in situations in which there is a high level of interdependence among teams (Joshi, Pandey, \& Han, 2009). When an organization is using a multiple-team membership structure and there are average to high interdependencies among these teams then a coordination mechanism needs to be in place in order to facilitate performance of these teams.

A leader displaying boundary spanning behaviors can facilitate information transfer and learning across teams and smoothen the coordination across teams. This can further have an impact on individual level outcomes (such as satisfaction with various memberships, level of stress, learning), as well as team level outcomes (such as cohesion, creativity or effectiveness). Suggestions of research questions here are:

New forms of organizing (such as multiple-team membership) might require new forms of leadership; are traditional forms of leadership (such as transactional, transformational) still relevant in these contexts? And if so, in what ways?

Which forms of leadership are the most suitable in order to maintain the performance and well-being of individuals and teams operating in a multiple-team membership setting?

What is the role of the boundary spanning leader in a multiple-team membership setting? Does task interdependence (across teams) play a role here?

\section{Multiple-team membership and time structuring}

One interesting aspect connected with the concept of multiple team membership is time structuring. When employees are part of one team at a time, they are able to focus on the tasks that need to be performed in this team. However, when they are part of multiple teams at the same time they need to be equipped with a time management scheme that allows them to divide their time across various teams and manage the number of switches across teams in an effective way. Switches for examples have been associated with attentional (and working memory) costs (Altmann \& Gray, 2008). After a task shift, cognitive mappings belonging to a previous task are still active and interfere with the task at hand, producing time delays and errors (Allport, Styles \& Hsieh, 1994).

Main research questions that emerge here are:

What is a good allocation of time across teams in order to enhance performance and learning, at individual, team and organizational level?

$\square$ What are antecedents of time structuring in a multiple-team membership setting: what is the role of leadership here?

Is there a managerial system or a technological system that can facilitate the management of time at an individual, organizational and team level? 


\section{Multiple team-membership and the idea of transfer}

To be noted that the phenomenon of multipleteam membership involves working with different levels of analysis. Individuals are nested within multiple teams and teams are embedded in one or more organizations. Such nested structures allow for information and resources flow across levels that can benefit individuals, teams and the whole organization. It would be definitively relevant to investigate in this setting how knowledge and information is transferred from one setting to another and what are the factors that facilitate an effective transfer. A few interesting research questions would be:

Do individuals benefit in terms of learning from the various teams they are part of? Does team variety matter here such that the higher the variety of teams (in terms of knowledge, tasks to be performed) an employee is part of, the higher the benefits?

Can we transpose the concept of multiple-team membership at higher levels of analysis, such as multipleorganization membership? What would be the similarities and the differences between the two concepts?

\section{Multiple-team membership and methodology}

Finally, it is important to acknowledge a few good methodological practices when studying the phenomenon of multiple-team membership.

Multiple team membership is already a context that implies some levels of complexity, requiring oftentimes multi-level analysis; for this reason, it is advisable to keep the design and the models simple in order to be able to identify robust results; more complexity does not necessarily mean better quality of results;

$\square \quad$ Use of designs that allow for causal inferences; it is essential to identify causal relations between variables in order to establish findings in a particular research field; the experimental design is a good candidate here;

Avoid self-reports measures when it comes to variables that can be measured in more objective ways, for example performance; self-reports are inaccurate, prone to biases and hence lead to inaccurate conclusions and accumulation of knowledge;

Studying multiple-team membership in an experimental fashion can be facilitated by the use of technology; finding adequate research platforms that facilitate the study of the phenomenon in an accurate manner is recommended.

\section{References}

Altmann, E. M., \& Gray, W. D. (2008). An integrated model of cognitive control in task switching. Psychological Review, 115(3), 602.

Allport, A., Styles, E. A., \& Hsieh, S. (1994). Shifting intentional set: Exploring the dynamic control of tasks. In C. Umiltà \& M. Moscovitch (Eds.), Attention and performance XV (pp. 421-452). Cambridge, MA: MIT Press.

Chen, G., Smith, T. A., Kirkman, B. L., Zhang, P., Lemoine, G. J., \& Farh, J. L. (2019). Multiple team membership and empowerment spillover effects: Can empowerment processes cross team boundaries? Journal of Applied Psychology, 104, 321-340. doi:10.1037/ap10000336

Cummings, J. N., \& Haas, M. R. (2012). So many teams, so little time: Time allocation matters in geographically dispersed teams. Journal of Organizational Behavior, 33, 316-341. doi:10.1002/job.777

Joshi, A., Pandey, N., \& Han, G. (2009). Bracketing team boundary spanning: An examination of task-based, team-level, and contextual antecedents. Journal of Organizational Behavior, 30, 731-759.

O’Leary, M. B., Mortensen, M., \& Woolley, A. W. (2011). Multiple team membership: A theoretical model of its effects on productivity and learning for individuals and teams. Academy of Management Review, 36, 461-478. doi:10.5465/ amr.2009.0275

Margolis, J. (2020). Multiple team membership: An integrative review. Small Group Research, 51(1), 4886.

Rapp, T. L., \& Mathieu, J. E. (2019). Team and individual influences on members' identification and performance per membership in multiple team membership arrangements. Journal of Applied Psychology, 104, 303-320. doi:10.1037/ ap10000344 